\title{
Synthesis, structural and electrical properties of novel pyrochlores in the $\mathrm{Bi2O3-CuO-}$ Ta2O5 ternary system.
}

\begin{abstract}
A series of non-stoichiometric cubic pyrochlores with general formula, $\mathrm{Bi3}-\mathrm{xCu} 1.8 \mathrm{Ta} 3+\mathrm{xO} 13.8+\mathrm{x}(\mathrm{BCT})$ was successfully prepared by solid state reaction at the firing temperature of $950{ }^{\circ} \mathrm{C}$ over 2 days. The solid solution mechanism is proposed as oneto-one replacement of $\mathrm{Bi} 3+$ for $\mathrm{Ta} 5+$, together with a variation in oxygen content in order to achieve electroneutrality. The solid solution limit is confirmed by X-ray diffraction technique (XRD) for which linear variation of lattice constants is observed at $0 \leq \mathrm{x} \leq 0.6$. The refined lattice constants are found to be in the range of 10.4838 (8) $\AA-10.5184$ (4) $\AA$ and the grain sizes of these samples determined by scanning electron microscopy (SEM) fall between 1 and $40 \mu \mathrm{m}$. Meanwhile, thermal analyses show no physical or chemical change for the prepared pyrochlores. The relative densities of the densified pellets for AC impedance measurements are above $85 \%$ and the measured relative permittivity, $\varepsilon^{\prime}$ and dielectric loss, $\tan \delta$ for composition, $\mathrm{x}=0.2$ at ambient temperature are $\sim 60$ and 0.07 at $1 \mathrm{MHz}$, respectively. The calculated activation energies are $0.32-0.40 \mathrm{eV}$ and the conductivity values, $\mathrm{Y}^{\prime}$ are in the order of $10-3$ at $400{ }^{\circ} \mathrm{C}$. The conduction mechanisms of BCT pyrochlores are probably attributed to the oxygen non-stoichiometry and mixed valency of copper within the structure.
\end{abstract}

Keyword: Solid state reaction; X-ray methods; Electrical properties; Tantalates. 